\title{
Protocolo de Higiene Oral para Establecimientos de Larga Estadía para Adultos Mayores en Estado de Pandemia COVID-19. Prevención de Neumonía por Aspiración
}

\author{
Oral Hygiene Protocol for Long-Term Establishments for Older Adults \\ in a State of Pandemic COVID-19. Aspiration Pneumonia Prevention
}

Francisco Díaz Méndez \& Julio Huerta Fernández²

DíAZ, M. F. \& HUERTA, F. J. Protocolo de higiene oral para establecimientos de larga estadía para adultos mayores en estado de pandemia COVID-19. Prevención de neumonía por aspiración. Int. J. Odontostomat., 14(4):508-512, 2020.

RESUMEN ¿Por qué es importante la salud oral en la prevención de enfermedades respiratorias en tiempos de pandemia? La razón es simple, cualquier enfermedad respiratoria en la persona mayor, podría eventualmente activar alarma de COVID-19 positivo, pudiendo ser esta ocasionada por otro motivo, sin mencionar el riesgo aumentado de tener una enfermedad previa de tipo respiratoria en tiempos de pandemia, sobre todo, en una población de riesgo como las personas mayores institucionalizadas. Por esta razón, la prevención en higiene oral de personas mayores mediante un Protocolo que proponga lineamientos a seguir en Establecimientos de Larga Estadía para Adultos Mayores, es fundamental en la prevención de Neumonía por aspiración. Cabe mencionar, que desde antes de surgir el COVID-19, ya la Neumonía se considera la principal causa de muerte por enfermedades infecciosas en la población chilena, y la primera causa específica de muerte en los mayores de 80 años.

PALABRAS CLAVE: persona mayor, establecimientos de larga estadía para adultos mayores, neumonía por aspiración, COVID-19, protocolo de higiene oral.

\section{INTRODUCCIÓN}

La familia de Coronavirus, comprende una variedad de virus que pueden causar enfermedades de diferente gravedad, tales como el MERS-COV o el SRAS-COV, que provoca el síndrome respiratorio agudo severo.

En los casos más graves, estos virus pueden llegar a causar Neumonía, e incluso, la muerte (OMS, 2020a).

En el último tiempo, se ha descubierto un nuevo Coronavirus, denominado COV-19, el cual era desconocido hasta su brote en China en 2019.

Actualmente, el mundo vive en un estado de pandemia ocasionado por COVID-19, infección en la que alrededor del $80 \%$ de las personas se recuperan sin necesitar hospitalización, sin embargo, existen grupos de riesgo como las personas mayores y las que padecen enfermedades sistémicas previas pudiendo presentar cuadros más graves, incluidos la Neumonía, pudiendo llegar a la muerte (OMS, 2020b).

Considerando que en las personas mayores institucionalizadas en Establecimientos de Larga Estadía para Adultos Mayores (ELEAM), una pobre higiene oral se muestra como uno de los factores de riesgo más comunes de Neumonía por aspiración (Quagliarello et al., 2005), se hace imprescindible disminuir todos los factores de riesgo mediante un protocolo de higiene oral rigurosa (Tada \& Miura, 2012) a fin de no empeorar o distinguir la Neumonía por COVID-19 de la causada por aspiración. El objetivo de este trabajo es presentar un Protocolo de higiene oral que puede ser aplicado en

\footnotetext{
${ }^{1}$ Cirujano Dentista Universidad Finis Terrae, Dip. Odontogeriatría Universidad de Chile, Diplomado en Geriatría y gerontología, INTA, Universidad de Chile. Práctica Privada en Odontogeriatría, Santiago, Chile.

${ }^{2}$ Cirujano Dentista Universidad de Concepción, Máster en Gerontología Social Universidad de León, Profesor Asociado, Universidad Finis Terrae, Santiago Chile.
} 
DÍAZ, M. F. \& HUERTA, F. J. Protocolo de higiene oral para establecimientos de larga estadía para adultos mayores en estado de pandemia COVID-19. Prevención de neumonía por aspiración. Int. J. Odontostomat., 14(4):508-512, 2020.

ELEAM por profesionales o personal auxiliar, con el fin de disminuir los riesgos de enfermedades respiratorias como la Neumonía por aspiración.

\section{Neumonía por Aspiración}

Se han encontrado bacterias orales en los pulmones de pacientes con Neumonía por aspiración (Bartlett et al., 1974). Los microorganismos de la cavidad oral, además, pueden comprometer los pulmones en personas de riesgo, como las personas mayores, debido a la aspiración de bacterias presentes en la enfermedad periodontal, las cuales, al entrar por las vías respiratorias provocan Neumonía, generando graves daños en la salud de la persona mayor, especialmente, a los que se encuentran en estado de fragilidad (Martínez Pascual et al., 2000).

De hecho, en un estudio en Japón se tomaron dos grupos de personas mayores en ELEAM. Luego, se realizó una comparación entre los que recibían y los que no recibían medidas de higiene oral especiales. Concluyeron que de los pacientes que contraían Neumonía, el $80 \%$ de ellos fallecía, al contrario de los que sí recibían cuidados orales, donde este porcentaje bajaba aproximadamente a la mitad (Yoneyama et al., 2002).

Es importante mencionar, que la Neumonía por aspiración suele darse en personas mayores y pacientes con problemas neurodegenerativos, siendo la edad y la mala higiene oral factores de riesgo para la colonización bacteriana orofaríngea (Carrillo-Náñez et al., 2013).

Cuando un paciente presenta otras comorbilidades (accidente cerebro vascular, disfagia, reflejo gastroesofágico) el riesgo de complicaciones respiratorias aumenta (Brunetti-Montenegro, 2013).

Sumado a lo anterior, es importante mencionar que la lengua de la persona mayor sufre cambios en su fuerza y coordinación, tendiendo a disminuir con el avance de los años, además del uso de ciertos medicamentos, los cuales, pueden provocar efectos secundarios como movimientos involuntarios, dificultando la alimentación y aumentando el riesgo de aspiración de fluidos o alimentos (Roisinblit, 2010).

\section{Descripción de Protocolo}

De acuerdo con la información expuesta, y la situación de pandemia que impera en el momento, con el fin de disminuir riesgos, se recomienda realizar la higiene oral con apoyo de un profesional o auxiliar entrenado, de esta manera, las personas mayores podrán lograr una mejor higiene oral, disminuyendo, por tanto, el riesgo de Neumonía por aspiración.

\section{Prevención de Infecciones}

Antes de realizar cualquier acción de higiene, tanto de la cavidad oral como protésica, el personal a cargo deberá efectuar lavado de manos por 40 a 60 segundos con agua y jabón, en su ausencia, y si las manos se encuentran limpias, usar un desinfectante de manos con un porcentaje de alcohol mínimo del $60 \%$ (alcohol gel), y posteriormente frotar por 20 segundos o hasta que se encuentren secas (Servicio Nacional del Adulto Mayor et al. , 2020).

Posteriormente, utilizar guantes de protección, mascarilla quirúrgica, pechera, lentes o protector facial. Todo esto con el fin de evitar tener contacto con la saliva. El uso de mascarilla quirúrgica es suficiente protección dado que el aseo de mucosas y lavado de dientes, no se incluyen dentro de las acciones liberadoras de aerosoles según la Orientación para la Atención Odontológica en Fase IV Covid-19 (Bartoszko et al., 2020; Ministerio de Salud, 2020a).

En el caso de realizar el aseo oral en el baño, limpiar las superficies con solución de cloro, en relación 1 litro de agua por 20 cc de cloro (4 cucharaditas), y utensilios con una solución de hipoclorito al 0,5\% Servicio Nacional del Adulto Mayor et al. , 2020; Ministerio de Salud, 2020b). De acuerdo con la estabilidad de SARS Cov-2 (COVID-19) se mantiene en acero y el plástico durante 7 días, y en el vidrio hasta 4 días (Chin et al., 2020), sería recomendable limpiar vasos plásticos, mangos de cepillos dentales (sin tocar los filamentos) y tubos de pasta dental, con solución de cloro diluida al 0,5\%.

Después del uso de cepillos de dientes, estos deben enjuagarse con abundante agua, ser almacenados en posición vertical y dejar secar al aire libre. De igual forma, las cajas de prótesis, deben ser almacenadas en un lugar fresco y seco (NHS, 2013). Se recomienda la limpieza de cajas de prótesis diariamente con la solución clorada.

Se debe cambiar el cepillo dental después de cualquier enfermedad contagiosa (Chin et al., 2012) 


\section{Posición para la Higiene Oral}

Las personas mayores que puedan estar erguidas, pueden mantener esa posición para realizar el cepillado asistido tanto de pie como sentadas. En el caso de personas en cama o con problemas de movilidad, el paciente debe ubicarse en posición semisentado, con la cabeza siempre inclinada hacia uno de sus lados en todo momento (Sumi, 2004). Se recomienda fijar al paciente en la posición con almohadas o toallas, con el fin de evitar que pueda quedar boca arriba, situación que puede favorecer la aspiración del contenido oral.

\section{Técnica de Cepillado Asistido}

Se recomienda lubricar los labios con bálsamo labial antes del cepillado, ya que los pacientes mayores suelen tener labios secos, los cuales, al momento de realizar el cepillado, pueden herirse y sangrar.

Si el paciente no puede mantener la boca abierta por el tiempo que dura el cepillado, puede utilizarse un baja lenguas esterilizado envuelto en gasa estéril, esto permitirá generar el espacio para realizar el cepillado dental, descansando la mordida y mejorando la experiencia del cepillado. En el caso de tener problemas para separar el labio de la encía se puede utilizar un baja lenguas untado en bálsamo labial, o el mango de un cepillo de dientes extra de propiedad de la misma persona mayor.

Al realizar el cepillado de dientes en personas dependientes, se puede ingresar el cepillo dental ubicándose de frente al paciente, o bien, posicionándose por detrás, apoyando la cabeza del paciente en el brazo del operador, y con la otra mano se manipula el cepillo dental con movimientos circulares.

Para realizar la higiene de dientes naturales, el cepillo debe tener un cabezal pequeño, también son de ayuda cepillos eléctricos si existe la posibilidad de disponer de ellos (NHS, 2013). El uso de gasa es un buen método para quitar los residuos orales y limpieza de mucosas en personas mayores que no poseen dientes naturales.

Abe et al. (2008), demostraron que en personas con ausencia de dientes naturales, la presencia de una lengua saburral es un indicador de riesgo para Neumonía. Por lo que la higiene oral debe ser rigurosa tanto en pacientes con dientes naturales como en edéntulos.

La gasa puede utilizarse enrollándola en el dedo enguantado de la persona que asiste en la higienización de la persona mayor. Para pacientes que suelen morder, es recomendable mantener la boca abierta con un baja lenguas envuelto en gasa o un mango de cepillo de dientes, para posteriormente limpiar los residuos con un baja lenguas envuelto en gasa. Es importante incluir todas las superficies, dientes, lengua y paladar. Sin olvidar la higiene interproximal, que se logra con mayor facilidad cuando existe el espacio suficiente con cepillos interproximales. El cepillado debe realizarse por un tiempo de mínimo 1 minuto, después de cada comida (Albert \& Lim, 2018).

\section{Uso de Pastas Dentales}

Las pastas dentales deben ser fluoradas, sin utilizar agua en el cepillo de dientes, el paciente no necesita escupir, o muchas veces no puede escupir, de esta forma el flúor se mantendrá en boca por más tiempo. El tamaño de aplicación de la pasta dental debe ser del tamaño de una arveja (Ministerio de Salud, 2015). Como recomendación, si existen problemas graves de deglución, se debe retirar de inmediato el exceso de espuma con una gasa y comunicarse con el odontólogo.

Luego de terminar con la higiene oral, se debe untar los labios de la persona mayor con bálsamo labial (Gil-Montoya et al., 2006).

\section{Cuidado de Prótesis Removibles}

Las prótesis removibles, deben retirarse durante la noche y guardarse en un lugar fresco y seco, de hecho, un estudio muestra que el uso de prótesis removibles durante la noche aumenta al doble el riesgo de Neumonía en las persona mayores en edad avanzada (Linuma et al., 2015). Deben lavarse al menos 2 veces al día, con jabón líquido neutro y escobilla de uñas, y finalmente, enjuagar con abundante agua. Para la desinfección semanal de prótesis acrílicas se debe sumergir en una solución de Hipoclorito de Sodio al 0,5\% por 3 minutos (Ministerio de Salud, 2019) y enjuagar con abundante agua. En el caso de que la prótesis tenga una base metálica se debe realizar inmersión en alcohol al $70 \%$ durante 5 minutos y posterior enjuague con agua (Consejo General de Colegios de Odontólogos y Estomatólogos de España, 2020), se recomienda realizarlo una vez por semana. La limpieza de prótesis es de suma importancia, ya que se ha documentado que la higienización poco frecuente de prótesis removibles puede aumentar el riesgo de Neumonía (Kusama et al., 2019). 


\section{CONCLUSIÓN}

El cuidado oral de la persona mayor institucionalizada debe ser tomado en cuenta como una prioridad dentro de las actividades diarias que se realizan en un ELEAM, considerando las graves implicancias que tiene para la persona mayor el estar expuesta a riesgos adicionales como la Neumonía por aspiración. Y más aún, en el escenario actual de pandemia, es que deben tomarse medidas en favor de la disminución de riesgos, para tales efectos un Protocolo de acción entrega un lineamiento a seguir en este difícil momento, contribuyendo finalmente con el cuidado y protección de la persona mayor.

DÍAZ, M. F. \& HUERTA, F. J. Oral Hygiene Protocol for LongTerm Establishments for Older Adults in a State of Pandemic COVID-19. Aspiration Pneumonia Prevention. Int. J. Odontostomat., 14(4):508-512, 2020.

ABSTRACT: Why is oral health important in the prevention of respiratory diseases in times of pandemic? The reason is simple, any respiratory disease in the elderly could eventually trigger a positive COVID-19 alarm, and this could be caused by another reason, not to mention the increased risk of having a previous respiratory-type illness in times of pandemic, over everything, in a population of risk like the institutionalized older people. For this reason, the prevention of oral hygiene in older people through a Protocol that proposes guidelines to be followed in long-stay establishments for older adults, is essential in the prevention of aspiration Pneumonia. It is worth mentioning that, before the emergence of COVID-19, Pneumonia was considered the main cause of death from infectious diseases in the Chilean population and the first specific cause of death in those over 80 years of age.

KEYWORDS: aged, homes for the aged, aspiration pneumonia, COVID-19, guideline for oral health.

\section{REFERENCIAS BIBLIOGRÁFICAS}

Abe, S.; Ishihara, K.; Adachi, M. \& Okuda, K. Tongue-coating as risk indicator for aspiration pneumonia in edentate elderly. Arch. Gerontol. Geriatr., 47(2):267-75, 2008.

Albert, M. \& Lim, W. T. Basic oral care for patiensts with dysphagia - Aspecial needs dentistry perspecticve. J. Clin. Pract. Speech Lang. Pathol., 20(3):142-9, 2018.

Bartlett, J. G.; Gorbach, S. L. \& Finegold, S. M. The bacteriology of aspiration pneumonia. Am. J. Med., 56(2):202-7, 1974.

Bartoszko, J. J.; Farooqi, M. A. M.; Alhazzani, W. \& Loeb, M. Medical masks vs N95 respirators for preventing COVID-19 in healthcare workers: a systematic review and meta-analysis of randomized trials. Influenza Other Respir. Viruses, 2020. DOI: https://www.doi.org/10.1111/irv.12745, 2020
Brunetti-Montenegro, F. Odontogeriatria uma Visao Gerontológica. Rio de Janeiro. Elsevier, 2013.

Carrillo-Ñáñez, L.; Muñoz-Ayala, M. P. \& Carrillo-García, P. L. Neumonía aspirativa en pacientes adultos mayores. Rev. Soc. Peru Med. Interna, 26(2):71-8, 2013.

Chin, A. W. H.; Chu, J. T. S.; Perera, M. R. A.; Hui, K. P. Y.; Yen, H. L.; Chan, M. C. W.; Peiris, M. \& Poon, L. L. M. Stability of SARS-CoV-2 in different environmental conditions. Lancet Microbe, 1(1):E10, 2020.

Chin, M.; Fenton, S. J.; Lyons, R.; Miller, C.; Perlman, S. P. \& Tesini, D. Dental Care Every Days. A Caregiver's Guide. Bethesda, U.S. Department of Health and Human Services, National Institutes of Health, National Institute of Dental and Craniofacial Research, 2012. Disponible en: https:// www.nidcr.nih.gov/sites/default/files/2017-09/dental-careevery-day-caregiver.pdf

Consejo General de Colegios de Odontólogos y Estomatólogos de España. Plan Estratégico de Acción para el Periodo Posterior a la Crisis Creada por el COVID-19. Madrid, Consejo General de Colegios de Odontólogos y Estomatólogos de España, 2020. Disponible en: https://www.consejodentistas.es/ comunicacion/actualidad-consejo/notas-de-prensa-consejo/ item/1763-plan-estrategico-de-accion-para-el-periodo-posterior-a-la-crisis-creada-por-el-covid-19.html

Gil-Montoya, J. A.; de Mello, A. L. F.; Cardenas, C. B. \& Lopez, I. G. Oral health protocol for the dependent institutionalized elderly. Geriatr. Nurs., 27(2):95-101, 2006.

Kusama, T.; Aida, J.; Yamamoto, T.; Kondo, K. \& Osaka, K. Infrequent denture cleaning increased the risk of pneumonia among community-dwelling older adults: a population-based cross-sectional study. Sci. Rep., 9(1):13734, 2019.

Linuma, T.; Arai, Y.; Abe, Y.; Takayama, M.; Fukumoto, M.; Fukui Y.; Iwase, T.; Takebayashi, T.; Hirose, N.; Gionhaku, N.; et al. Denture wearing during sleep doubles the risk of pneumonia in the very elderly. J. Dent. Res., 94(3 Suppl.):28S-36S, 2015.

Martínez Pascual, A.; Estany Castella, J. \& Vallcorba Plana, N. Neumonia por aspiración y enfermedad periodontal. Periodoncia, 10(2):105-14, 2000.

Ministerio de Salud (MINSAL). Guía Clínica AUGE. Subsecretaría de Salud Pública División de Prevención y Control de Enfermedades Departamento de Salud Bucal Salud Oral Integral para Adultos de 60 años: Prevención y Tratamiento de Caries Radiculares. Santiago de Chile, Ministerio de Salud, Gobierno de Chile, 2015. Disponible en: http:// www.bibliotecaminsal.cl/wp/wp-content/uploads/2016/04/Salud-Oral-Integral-para-adultos-de-60-a\% C3\%B1 osradicular.pdf

Ministerio de Salud (MINSAL). Orientación para la Atención Odontológica en Fase IV Covid-19. Santiago de Chile, Ministerio de Salud, Gobierno de Chile, 2020a. Disponible en: https:/ /diprece.minsal.cl/wp- content/uploads/2020/03/ORIENTACIONES-ATENCION- ODONTOLOGICAS-COVID-19-.pdf

Ministerio de Salud (MINSAL). Protocolo de Limpieza y Desinfección de Ambientes. Santiago de Chile, Ministerio de Salud, Gobierno de Chile, 2020b. Disponible en: https://www.minsal.cl/ wp-content/uploads/2020/03/PROTOCOLO-DE-LIMPIEZAYDESINFECCI\%C3\%93N-DE-AMBIENTES-COVID-19.pdf

Ministerio de Salud (MINSAL). Recomendaciones de Higiene Bucal y Cuidados para Personas Portadoras de Prótesis Dentales Removibles. Santiago de Chile, Ministerio de Salud, Gobierno de Chile, 2019. Disponible en: https://diprece.minsal.cl/wpcontent/uploads/2019/05/RECOMENDACIONES-DE-HIGIENE-Y-CUIDA-DOS-PARA-PERSONAS-PORTADORAS-DEPR\%C3\%93TESIS-REMOVIBLES-24042019.pdf

NHS Health Scotland. Caring for Smiles. Guide for Care Homes. Better Oral Care for Dependent Older People. Edinburgo, NHS 
Health Scotland, 2013. Disponible en: http:// www.knowledge.scot.nhs.uk/media/7460397/ caringforsmilescarehomes2013.pdf

Organización Mundial de la Salud (OMS). Brote de Enfermedad por Coronavirus (COVID-19). Ginebra, Organización Mundial de la Salud, 2020b. Disponible en: https://www.who.int/ es/emergencies/diseases/novel-coronavirus-2019

Organización Mundial de la Salud (OMS). Coronavirus. Ginebra, Organización Mundial de la Salud, 2020a. Disponible en: https://www.who.int/es/health-topics/coronavirus

Quagliarello, V.; Ginter, S.; Han, L.; Van Ness, P.; Allore, H. \& Tinetti, M. Modifiable risk factors for nursing home-acquired pneumonia. Clin. Infect. Dis., 40(1):1-6, 2005.

Roisinblit, R. Odontología para las Personas Mayores. Buenos Aires, Edición del Autor, 2010.

Servicio Nacional del Adulto Mayor; Departamento de Ciclo Vital, de la División de Prevención y Control de Enfermedades del Ministerio de Salud \& Sociedad de Geriatría y Gerontología de Chile. Fases 3 y 4:Protocolo de recomendaciones para la Prevención y Atención del COVID-19 en Centros Residenciales, Ambulatorios y Clubes de Adultos Mayores. Santiago de Chile, Ministerio de Salud, Ministerio de Desarrollo Social y Familia, Gobierno de Chile, Sociedad de Geriatría y Gerontología de Chile, 2020. Disponible en: http:// w w w. s e n a ma.gob.cl/storage/docs/. $\mathrm{Fases} 3$ y 4 Protocolo COVID. 19_y_Personas_Mayores_-002.pdf

Sumi, Y. Oral Care for the Dependent Elderly. Oral Care System. Oral Care Support Instrument. Obu, National Center for Geriatrics and Gerontology, 2004. Disponible en: https:// www.ncgg.go.jp/hospital/english/clinics/documents/ oralcavitycare en1.pdf

Tada, A. \& Miura, H. Prevention of aspiration pneumonia (AP) with oral care. Arch. Gerontol. Geriatr., 55(1):16-21, 2012.

Valdivia, C. G. Epidemiología de la neumonía del adulto adquirida en la comunidad. Rev. Chil. Infectol., 22(Supl. 1):S11S17, 2005.

Yoneyama T.; Yoshida, M.; Ohrui, T.; Mukaiyama, H.; Okamoto, H.; Hoshiba, K.; Ihara, S.; Yanagisawa, S.; Ariumi, S.; Morita, T.; et al. Oral care reduces pneumonia in older patients in nursing homes. J. Am. Geriatr. Soc., 50(3):430-3, 2002.
Dirección para correspondencia:

Dr. Francisco Díaz Méndez

Cirujano Dentista

Universidad Finis Terrae

Santiago

CHILE

Email: nicofin2q@gmail.com

Recibido : 25-05-2020

Aceptado: 01-06-2020 